\title{
Über den Unterschied zwischen theoretischen und practischen Zinsrechnungen.
}

(Nachtrag zu der Abhandlung über Sparcassen im 39. Bande dieses Journals S. 183 etc.)

$$
\text { (Vom Herausgeber.) }
$$

1.

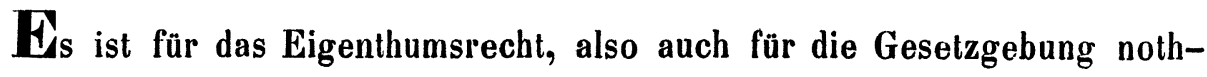
wendig, den Unterschied der Ergebnisse theoretischer und practischer Zinsrechnungen zu berücksichtigen. Einen solchen, nicht unbedeutenden Unterschied giebt es, wie sich zeigen wird, wirklich.

Es sind insbesondere die beiden Fragen zu untersuchen:

Erstlich. Bis zu welchem Betrage eine bestimmte Summe in einer gewissen Zahl von Jahren durch ihre Zinsen anwächst, und

Zweitens. Welche Summe durch eine jührliche oder halbjährliche Zahlung und durch ihre Zinsen in einer bestimmten Zahl von Jahren aufgehäuft wird.

Diese Fragen kommen häufig in der Wirklichkeit vor. Z. B. bei RentenAblösungen, Tilgung von Anleihen, Renten- und Wittwencassen etc., und überall wo ein Interusurium oder gesetzliches Disconto in Betracht kommt. Die Erörterung der Ergebnisse wird sich leicht auch auf etwanige andere Fragen anwenden lassen.

2.

Man kann, aus diesem oder jenem Grunde, entweder blofs einfache Zinsen, wie Hoffinann und Andere, oder auch noch Zinseszinsen, wie z. B. Leibnitz, in Rechnung bringen. In beiden Fällen ist die theoretische Beantwortung der obigen beiden Fragen sehr leicht. Aber die Ergebnisse treffen in beiden Fällen practisch nicht das Rechte, und die Abweichung vom Richtigen kann, besonders bei kleinen Summen, verhältni/smä/sig sehr bedeutend sein. Man setzt nämlich voraus, dafs sich jede, auch die kleinste Summe, jeden Augenblick und ohne Schwierigkeit und Mühe zinsbar sicher anlegen lasse; was bekanntlich in der Wirklichkeit nicht der Fall ist. Für grö/sere Summen ist der Unterschied der theoretischen und praktischen 
Ergebnisse verhälıni/smä/sig wenig bedeutend, wie es sich an einigen Beispielen zeigen wird. Aber gerade besonders für kleine Summen ist es nöthig, dafs das Gesetz den Besitzer vor Schaden wahre, weil für Den, welcher Wenig hat, auch schon Wenig Viel sein kann.

3.

Als Zinsfufs wird gewöhnlich der Jahreszins angegeben und angenommen. Aber gezahlt werden Zinsen fast durchweg halbjährlich; besonders bei allen zinstragenden Papieren. Man wird daher in der Regel halbjälırliche Zinsen in Rechnung zu bringen haben.

$s$ sei eine bestimmte Zahl von Geld-Einheiten.

$n$ eine Zahl von halben Jahren.

$z$ sei der halbjährliche Zins von 1.

$\boldsymbol{X}$ sei die Summe, welche durch Hinzuthun einfacher Zinsen und

$x$ die natürlich kleinere Summe, welche durch Hinzuthun von einfachen und von Zinseszinsen in $n$ halben Jahren zu der Summe $s$ unter der Bedingung anwächst, dafs sich jede, auch die kleinste Summe, in jedem Augenblick zu dem Zinsfufs $\approx$ anlegen läfst.

$\boldsymbol{Y}$ sei eine halbjährliche gleiche Zahlung, welche durch Hinzuthun einfacher Zinsen und

y die, natürlich kleinere halbjährliche Zahlung, welche durch Hinzuthun von einfachen und Zinseszinsen in $n$ halben Jahre die Summe $s$ giebt; beides unter der obigen Bedingung.

$a$ sei die kleinste Summe, welche sich wirklich stets ohne Mühe und Schwierigkeit zu dem Zinsfufs $z$ sicher anlegen läfst.

$\boldsymbol{X}_{n}$ und $\boldsymbol{Y}_{n}$ seien die Summen, welche sich aus $\boldsymbol{X}$ und $\boldsymbol{Y}$ durch Hinzuthun einfacher Zinsen und

$x_{n}$ und $y_{n}$ diejenigen, welche sich aus $x$ und $y$ durch Hinzuthun von einfachen und von Zinseszinsen in $\boldsymbol{n}$ halben Jahren wirklich aufhänfen lassen; nämlich unter der Bedingung, dafs nur Vielfache von a auf Zinsen ausgethan werden können.

$\sigma$ sei die Summe und

$\varepsilon$ die halbjährliche Zahlung, welche in $n$ halben Jahren durch Hinzuthun von wirklich zu erlangenden Zinsen näherungsweise die Summe $s$ einträgt. 
$\sigma_{n}$ und $\varepsilon_{n}$ seien die Summen, bis zu welchen $\sigma$ und $\varepsilon$ in $n$ halben Jahren durch Hinzuthun von wirklich zu erlangenden Zinsen anwachsen, so dafs, wenn $\sigma$ und $\varepsilon$ genau berechnet werden, $\sigma_{n}$ und $\varepsilon_{n}$ gleich $s$ sind.

4.

Erstlich. Eine bestimmte Summe wächst durch Zinsen, theoretisch, wie folgt an.

I. Die Summe $X$ durch einfache Zinsen.

Da jedes halbe Jahr $\approx X$ zu $\boldsymbol{X}$ hinzukommt, so nimmt $\boldsymbol{X}$ in $n$ halben Jahren um $n z X$ und folglich bis zu $X+n z X$ zu. Soll also Dies die Summe $s$ geben, so mufs

(1.) $s=(1+n z) X$ sein, woraus

(2.) $\quad X=\frac{s}{1+n z}$ folgt; desgleichen

(3.) $n=\frac{s-X}{z X}$ und

(4.) $z=\frac{s-X}{n X}$.

II. Die Summe $x$ durch Zinseszinsen.

Da der Ertrag jedes halben Jahres $1+z$ mal so grofs wird, so ist der Ertrag von $x$ nach $n$ halben Jahren $x(1+z)^{n}$. Soll Dies die Summe $s$ geben, so mufs

$$
\begin{aligned}
& \text { (5.) } \quad s=x(1+z)^{n} \text { sein, woraus } \\
& \text { (6.) } \quad x=\frac{s}{(1+z)^{n}} \text { folgt; desgleichen } \\
& \text { (7.) } \quad n=\frac{\log s-\log x}{\log (1+z)} \text { und } \\
& \text { (8.) } \quad \log (1+z)=\frac{\log s-\log x}{n}
\end{aligned}
$$

Zweitens. Eine gleiche halbjährliche Zahlung trägt theoretisch Folgendes ein.

I. Die halbjährliche Zahlung $\boldsymbol{Y}$ durch einfache Zinsen.

Es werde angenommen, dafs, wie es gewöhnlich der Fall ist, die erste halbjährliche Zahlung am Ende des ersten, die letzte halbjährliche Zahlung am Ende des letzten halben Jahres erfolge. 
Dann ist die erste halbjährliche Zahlung $Y$, welche am Ende des ersten halben Jahres erfolgte, bis zum letzten halben Jahre, die $n-1$ halben Jahre hindurch, nach (1.), bis zu $[1+(n-1) z] Y$ angewachsen; die zweite halbjährliche Zahlung $\boldsymbol{Y}$, die $n-2$ halben Jahre hindurch, bis zu $[1+(n-2) z] Y$; die dritte halbjährliche Zahlung $Y$, die $n-3$ halben Jahre hindurch, bis zu $[1+(n-3) z] Y$ u.s. w. Die letzte halbjährliche Zahlung giebt blofs $\boldsymbol{Y}$. Also ist überhaupt aufgesammelt worden:

$$
\begin{aligned}
(1+2+3+4 \cdots+n-1): Y+n Y & =n Y+\frac{1}{2}(1+n-1)(n-1) z Y \\
& =n Y\left[1+\frac{1}{2}(n-1) z\right] .
\end{aligned}
$$

Soll Dies die Summe $s$ geben, so mufs

$$
\text { (9.) } s=n Y\left[1+\frac{1}{2}(n-1) z\right]
$$

sein, und daraus folgt

$$
\text { (10.) } \quad Y=\frac{s}{n\left[1+\frac{1}{2}(n-1) z\right]} \text {. }
$$

Ferner folgt aus (9.) $s=\frac{1}{2} n^{2} z Y-\frac{1}{2} n z Y+n Y$ oder $n^{2}-2 n\left(\frac{1}{2}-\frac{1}{z}\right)-\frac{2 s}{z Y}=0$, also $n=\frac{z-2}{2 z} \pm \sqrt{ }\left[\left(\frac{z-2}{2 z}\right)^{2}+\frac{2 s}{z Y}\right]$ oder

$$
\text { (11.) } n=\frac{z-2 \pm \sqrt{ }\left[(z-2)^{2}+\frac{8 s}{Y}\right]}{2 z} \text {. }
$$

Endlich folgt aus (9.)

$$
\text { (12.) } \quad z=\frac{2(s-n Y)}{n(n-1) Y} \text {. }
$$

II. Die halbjährliche gleiche Zahlung $\boldsymbol{Y}$ durch Zinseszinsen.

Die erste halbjährliche Zahlung $y$, welche am Ende des ersten halben Jahres erfolgt, wächst bis zum Ende des nten halben Jahres, die $n-1$ halben Jahre hindurch, nach (5.) bis zu $y(1+z)^{n-1}$ an; die zweite halbjährliche Zahlung $y$, die $n-2$ halben Jahre hindurch, bis zu $y(1+z)^{n-2}$; die dritte halbjährliche Zahlung, die $n-3$ halben Jahre hindurch, bis zu $y(1+z)^{n-3}$ u. s. w. Die letzte halbjährliche Zahlung $y$ thut blofs $y$ hinzu. Also ist überhaupt aufgesammelt worden: $\left[1+(1+z)+(1+z)^{2}+(1+z)^{3} \cdots+(1+z)^{n-1}\right] y=\frac{(1+z)^{n}-1}{1+z-1} \cdot y$ $=\frac{(1+z)^{n}-1}{z} \cdot y$. Soll Dies die Summe $s$ geben, so mufs

$$
\text { (13.) } s=\frac{(1+z)^{n}-1}{z} \cdot y
$$


sein. Hieraus folgt

$$
\text { (14.) } \quad y=\frac{z s}{(1+z)^{n}-1}
$$

desgleichen $s z+y=(1+z)^{n} y$, also $n \log (1+z)=\log \left(1+\frac{z s}{y}\right)$ und

endlich

$$
\text { (15.) } \quad n=\frac{\log (z s+y)-\log y}{\log (1+z)} \text {; }
$$

$$
\text { (16.) } \log (1+z)=\frac{\log (z s+y)-\log y}{n} \text {. }
$$

Aus der Gleichung (16.) läfst sich $z$ nur näherungsweise durch Versuche finden, wie es auch sonst häufig, z. B. bei der Auflösung algebraischer Gleichungen von höheren Graden vorkommt. Man kann für $z$ zunächst den Werth von $z$, welchen (12.) für einfache Zinsen und für $\boldsymbol{Y}=\boldsymbol{y}$ giebt und welcher etwas zu gro/s sein wird, rechts in (16.) setzen. Dann verkleinert man ihn allmälig, bis der Gleichung (16.) genug gethan wird. Die übrigen obigen Gleichungen lassen sich unmittelbar auflösen.

Man möge nun an ein Paar bestimmten Fällen sehen,. inwiefern die Ergebnisse der theoretischen Ausdrücke von denen in der Wirklichkeit abweichen.

5.

Frage No. 1. A hat die Nutznie/sung einer Summe $s$, welche $\operatorname{dem} \boldsymbol{B}$ gehört, auf $n$ halbe Jahre. Wieviel kann und mufs $B \operatorname{dem} \boldsymbol{A}$ am Anfange der $n$ halben Jahre zahlen, wenn er dem $\boldsymbol{A}$ die Nutzniefsung abkaufen will?

a. Offenbar so viel, dafs $B$ aus der Summe, welche er von $s$ übrig behrilt, in $n$ halben Jahren die ihm dann gebührende Summe $s$ aufhäufen kann. Die geringeren immerwährenden Zinsen aus dem Theile von $s$, welchen $A$ erhält, sind so viel werth als die vollen Zinsen von $s$ auf $n$ halbe Juhre.

b. Der jährliche Zinsfufs sei 4 vom Hundert, also

$$
\text { (17.) } z=0,02 \text {. }
$$

Ferner habe $\boldsymbol{A}$ die Nutzniefsung der Summe $s$ auf 20 Jahre, so dafs

$$
\text { (18.) } n=40
$$

ist. Und dann sei zunächst die Summe

$$
\text { (19.) } s=100 \text { Thaler. }
$$


c. Bringt man dem $B$ blofs einfache Zinsen in Rechnung, so ist Das, was er von den 100 Thalern übrig behalten mufs, nach (2.) theoretisch:

$$
X=\frac{s}{1+n z}=\frac{100}{1+40.0,02}=\frac{100}{1,8}=55,555 \text { Thaler, }
$$

und er hat also 44,444 Thaler $\operatorname{dem} A$ zu zahlen.

Bringt man dem $\boldsymbol{B}$ Zinseszinsen in Rechnung, so ist Das, was er von den 100 Thalern übrig behalten mufs, nach (6.):

(21.) $x=\frac{s}{(1+z)^{n}}=\frac{100}{1,02^{40}}=45,289$ Thlr. (durch Logarithmen berechnet), und er hat also dem $\boldsymbol{A}, 54,711$ Thaler zu zahlen.

d. Sicherlich würde nun $B$ am Ende des 20ten Jahres zu seinen 100 Thalern gelangen, wenn er halbjährlich 2 v. H. einfache Zinsen zu der Summe $X=55,555$ Thaler, oder 2 v. H. Zinsen und Zinseszinsen zu der Summe $x=45,289$ Thaler, die er übrig behält, hinzu legen könnte. Aber es ist nicht vorauszusetzen, dafs $B$ sogleich Jemand finden werde, der gerade 55,555 Thaler zu 4 v. H. jährlich zu leihen verlangt. Er wird ihn suchen müssen, und dieses Suchen erfordert, wenn nicht baare Kosten, so doch Zeit und Mühe; und Zeit und Mühe sind auch Geld. Findet er nur zu einer geringeren runden Summe, z. B. zu 50 Thalern, einen Schuldner, so verliert er schon die Zinsen von dem Rest. Es ist möglich, dafs ihm Dies durch die Zinseszinsen, welche er erlangen kann, wieder einkommt; allein es ist nicht sicher.

Entschieden ist sein Schaden, wenn er nur 45,289 Thaler übrig behält, und nun Zins vom Zins aufhäufen soll. Denn er mufs dann nicht blofs gleich Anfangs einen Schuldner suchen, welcher gerade 45,289 Thaler geliehen verlangt, sondern auch jedes halbe Jahr Scbuldner für die geringeren Zinsbeträge (z. B. von $0,02.45,289=0,90578$ Thaler nach dem ersten halben Jahr); was ihm schwerlich gelingen wird. Findet er nur für weniger Schuldner, so verliert er von den Zinsen; und finden sich nur Solche, die mehr verlangen, so mufs er von dem Seinigen zulegen; wozu er nicht verbunden ist.

e. Alles was $\boldsymbol{B}$ zu thun vermay, ist, dafs er für so viel als es angeht aus den $\boldsymbol{x}$ oder $\boldsymbol{X}$ Thalern, die er übrig behielt, so wie späterhin aus den aufgesammelten Zinsen, sichere zinstragende und ohne namhafte Mühe zu erlangende Papiere kauft, das Übrige aber, waś noch nicht zu den kleinsten käuflichen Papieren hinreicht, zinslos zu seinem Bestande hinzuthut.

$f$. Es wäre also zu berechnen, welche Summe $\boldsymbol{B}$ aus den Summen $X$ und $x$ die 40 halben Jahre hindurch in den beiden Fällen, wenn ihm nur 
einfache Zinsen und wenn ihm Zinseszinsen in Anrechnung gebracht werden, wirklich aufzuhäufen vermag.

Es werde angenommen, dafs, wie es in der Wirklichkeit meistens der Fall sein wird, das kleinste, überall käufliche sichere Zinspapier, welches 2 v. H. halbjährliche Zinsen trägt, auf

$$
\text { (22.) } \quad a=10 \text { Thaler }
$$

laute.

g. Dann sind die Bestände, zu welchen $\boldsymbol{B}$ allmälig gelangt, folgende. Wenn dem $B$ blo/s einfache Zinsen in Rechnung gebracht werden, also von $X=\mathbf{5 5 , 5 5 5}$ Thlrn.

\begin{tabular}{|c|c|c|c|c|c|c|c|}
\hline $\begin{array}{l}\text { Am Ende } \\
\text { des halben } \\
\text { Jahres. }\end{array}$ & Bestand. & $\begin{array}{l}\text { Nämlich } \\
\text { von } \\
\text { früher. }\end{array}$ & $\begin{array}{l}\text { Und an } \\
\text { Zins. }\end{array}$ & Von & Aus & & \\
\hline $\begin{array}{c}\text { Thlr. } \\
5\end{array}$ & $\begin{array}{c}\text { Thtr. } \\
60,555\end{array}$ & $\begin{array}{c}\text { Thlr. } \\
55,555\end{array}$ & $\begin{array}{r}\text { Thlir. } \\
5,0\end{array}$ & $\begin{array}{c}\text { Thlr. } \\
50\end{array}$ & 5 & halben & Jahren, \\
\hline 13 & 70,155 & 60,555 & 9,6 & 60 & 8 & - & - \\
\hline 21 & 81,355 & 70,155 & 11,2 & 70 & 8 & - & - \\
\hline 27 & 90,955 & 81,355 & 9,6 & 80 & 6 & - & - \\
\hline 33 & 101,755 & 90,955 & 10,8 & 90 & 6 & - & - \\
\hline 38 & 111,755 & 101,755 & 10,0 & 100 & 5 & - & - \\
\hline 48 & 116,155 & 111,755 & 4,4 & 110 & 2 & - & - \\
\hline
\end{tabular}

Also kann $B$, bis zum Ende des 20ten Jahres aus $X=55,555$ Thlrn. die Summe (24.) $X_{n}=116,155$ Thaler

wirklich aufhäufen; was über 16 v. H. mehr ist, als ihm zukommt. Folglich wird $\boldsymbol{B}$, wenn man ihm blo/s einfache Zinsen in Ansatz bringt, bedeutend begünstigt und $A$ gegenseits durch die Zahlung von blofs 44,444 Thlrn. benachtheiligt.

Wenn dem $B$ Zinsessinsen in Rechnung gebracht werden, also von $x=45,289$ Thlrn.

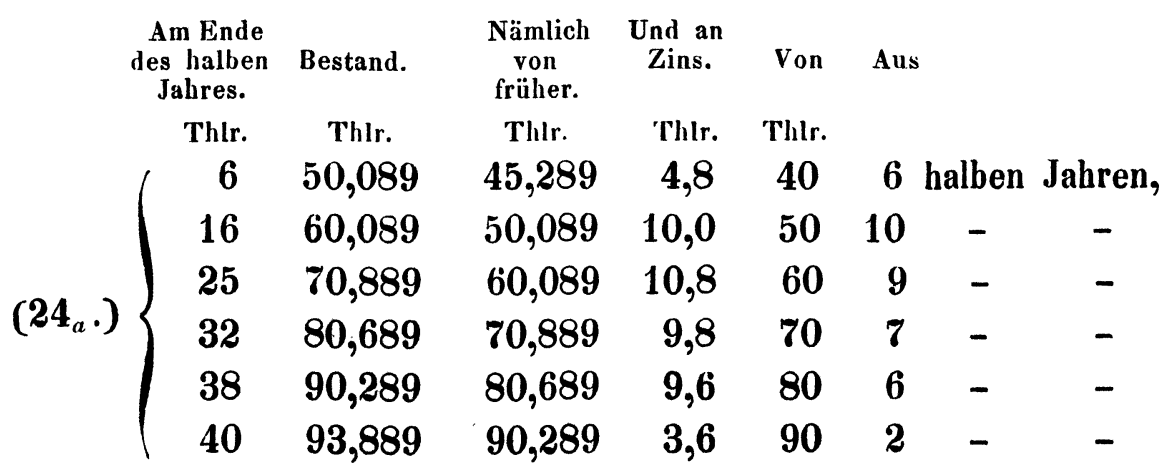


Also vermag $B$ bis zum Ende des 20ten Jahres aus $x=45,289$ Thalern nur die Summe

$$
\text { (25.) } x_{n}=93,889 \text { Thaler }
$$

aufzuhäufen, was über 6 v. H. weniger ist, als ihm zukommt. Folglich wird $\boldsymbol{B}$, wenn man ihm Zinseszinsen in Ansatz bringt, bedeutend benachtheiligt und dagegen $\boldsymbol{A}$ gegenseits durch die Zahlung von 54,711 Thlrn. begünstigt.

Mithin trifft weder die Anrechnung von blofs einfuchen Zinsen, noch die von Zinseszinsen das Rechte.

h. Bei grölseren Summen ist der Fehler bei der Anrechnung von Zinseszinsen verhältnifsmäfsig nur gering, bei der Anrechnung von blofs einfachen Zinsen aber noch viel gröfser.

Es sei z. B. $s$, statt wie vorhin 100 Thaler,

$$
\text { , (26.) } s=10000 \text { Thaler. }
$$

Dann sind $X$ und $x$ in (2. und 6.) 100 mal so grofs und folglich ist

(27.) $X=5555,5$ Thaler für blofs einfache Zinsen,

(28.) $x=4528,9$ Thaler für die Anrechnung von Zinseszinsen.

Wir wollen von der Rechnung, zunächst für einfache Zinsen, um den Raum zu sparen, blofs den Anfang bersetzen.

Wenn dern B blo/s einfache Zinsen in Ansatz gebracht, also 5555,5 Thlr. zugestanden werden, so sind die Beträge folgende:

Am Ende des halben Jahres.

Bestand.

Nämlich vom vorigen Jahre.

Und an Zins.

Von

1 Thlr. 5666,5 Thlr. 5555,5 Thlr. 111 Thlr. 5550 Thlr.

$2-5779,7-5666,5-112,2-5660-$

(29.)

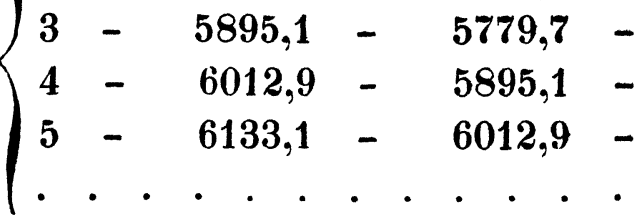

Das End-Ergebnifs ist

$$
\text { (30.) } \quad X_{n}=12260,3 \text { Thlr. }
$$

Also kann $B$ aus den ihm zu einfachen Zinsen berechneten 5555,5 Thlrn. in 20 Jahren nicht blofs 10000 Thlr., sondern 12260,3 Thlr. aufhäufen; er bekommt also über $22 \frac{1}{2}$ v. H. mehr, als thm gebührt. 
Werden dagegen dem $B$ Zinseszinsen in Anrechnung gebracht, so ergiebt eine ganz ähnliche Rechnung, dafs er von den ihm alsdann zugestandenen 4528,9 Thlrn. in 20 Jahren nicht 10000 Thlr., sondern nur

$$
\text { (31.) } \quad x_{n}=9993,3 \text { Thlr. }
$$

aufzuhäufen vermag, dafs er mithin 6,7 Thlr. zu wenig bekommt; was nicht bedeutend ist.

6.

Frage No. 2. $A$ hat dem $B$ eine immerwährende Rente zu zahlen, welche halbjährlich 2 v. H. der Summe $s$ beträgt. Wieviel mufs $A$ zu der Rente $n$ halbe Jahre lang halbjährlich zulegen, um sie abzulösen:

Oder auch:

$\boldsymbol{A}$ ist dem $\boldsymbol{B}$ die Summe $s$ schuldig, mufs sie ihm mit 2 v. H. halbjährlich verzinsen und zuletzt, wenn auch nach noch so langer Zeit, zurückzahlen. Wieviel mufs $\boldsymbol{A} \boldsymbol{n}$ halbe Jahre lang halbjährlich zu den Zinsen $\boldsymbol{z} u$ legen, um dann nach $n$ halben Jahren nichts zurückzahlen zu dürfen?

a. Offenbar so viel, dafs $\boldsymbol{B}$ aus der Zulage in $\boldsymbol{n}$ halben Jahren die Summe $s$ aufzuhäufen vermag, weil er dann weiterhin, statt von $\operatorname{dem} \boldsymbol{A}$, aus der Summe $s$ die ihm gebührende Rente, oder die ihm gebührenden vollen Zinsen zieht.

b. Die nöthige halbjährliche Zulage wird, wenn man dem $\boldsymbol{B}$ nur einfuche Zinsen in Ansatz bringt, die $\boldsymbol{Y}$ (10.), und wenn man ihm Zinseszinsen anrechnet, die $y$ (14.) sein.

c. Es sei nun zuerst

$$
\text { (32.) } s=100 \text { Thaler, } n=40 \text {, }
$$

so ergiebt sich aus (10.) und aus (14.):

(33.) $\quad Y=\frac{s}{n\left[1+\frac{1}{2}(n-1) z\right]}=\frac{100}{40(1+19,5.0,02)}=\frac{100}{55,6}$

$$
=1,7985 \text { Thlr. für einfache Zinsen und }
$$

(34.) $\quad y=\frac{z s}{(1+z)^{n}-1}=\frac{0,02.100}{1,02^{40}-1}=1,6555$ Thlr. für Zinseszinsen.

d. Es kommt jelzt wieder darauf an, welche Summe $\boldsymbol{B}$ aus diesen halbjährlichen Zahlungen in 20 Jahren aufzuhäufen vermag; wobei wie in (\$. 5. 22.)

angenommen werden soll.

$$
\text { (35.) } a=10 \text { Thaler }
$$


e. Die Beträge sind folgende:

Wenn dem $B$ blo/s einfache Zinsen in Rechnung gebracht werden, also aus der halbjährlichen Zahlung von 1,7985 Thlr. (33.):

\begin{tabular}{|c|c|c|c|c|c|c|c|c|}
\hline $\begin{array}{l}\text { Am Ende } \\
\text { des lialben } \\
\text { Jalires. }\end{array}$ & Bestand. & $\begin{array}{c}\text { Nämlich } \\
\text { von, } \\
\text { trüher. }\end{array}$ & $\begin{array}{l}\text { Und dazu } \\
\text { an halb- } \\
\text { âhrlichem } \\
\text { Zins. }\end{array}$ & Von & $\begin{array}{c}\text { Und an } \\
\text { halb- } \\
\text { jällilicher } \\
\text { Zahlung. }\end{array}$ & Aus & & \\
\hline $\begin{array}{c}\text { Thltr. } \\
6\end{array}$ & $\begin{array}{c}\text { Thlr. } \\
10,7710\end{array}$ & $\begin{array}{l}\text { Thlr. } \\
\end{array}$ & $\begin{array}{c}\text { Thlr. } \\
0\end{array}$ & $\begin{array}{c}\text { Thlr. } \\
0\end{array}$ & $\begin{array}{c}\text { Thlr. } \\
1,7985\end{array}$ & 6 & halben & Jahr \\
\hline 11 & 20,7635 & 10,7710 & 0,2 & 10 & 1,7985 & 5 & - & - \\
\hline 16 & 31,7560 & 20,7635 & 0,4 & 20 & 1,7985 & 5 & - & - \\
\hline 20 & 41,3500 & 31,7560 & 0,6 & 30 & 1,7985 & 4 & - & - \\
\hline 24 & 51,7400 & 41,3500 & 0,8 & 40 & 1,7985 & 4 & - & - \\
\hline 27 & 60,1355 & 51,7400 & 1,0 & 50 & 1,7985 & 3 & - & - \\
\hline 31 & 72,1295 & 60,1355 & 1,2 & 60 & 1,7985 & 4 & - & - \\
\hline 34 & 81,7250 & 72,1295 & 1,4 & 70 & 1,7985 & 3 & - & - \\
\hline 37 & 91,9210 & 81,7250 & 1,6 & 80 & 1,7985 & 3 & - & - \\
\hline 40 & 102,7165 & 91,9210 & 1.8 & 90 & 1,7985 & 3 & - & \\
\hline
\end{tabular}

Also vermag $B$ bis zum Ende des $20^{\text {ten }}$ Jahres aus der halbjährlichen Zahlung von 1,7985 Thlrn. die Summe

(37.) $\quad Y_{n}=102,7165$ Thlr.

aufzuhäufen, mithin nahe an 3 v. H. mehr, als ihm zukommt. Er wird daher durch die Anrechnung von blo/s einfachen Zinsen begünstigt.

Wenn dem $B$ Zinseszinsen in Rechnung gebracht werden; also aus der halbjührlichen Zahlung von 1,6555 Thlr. (34.):

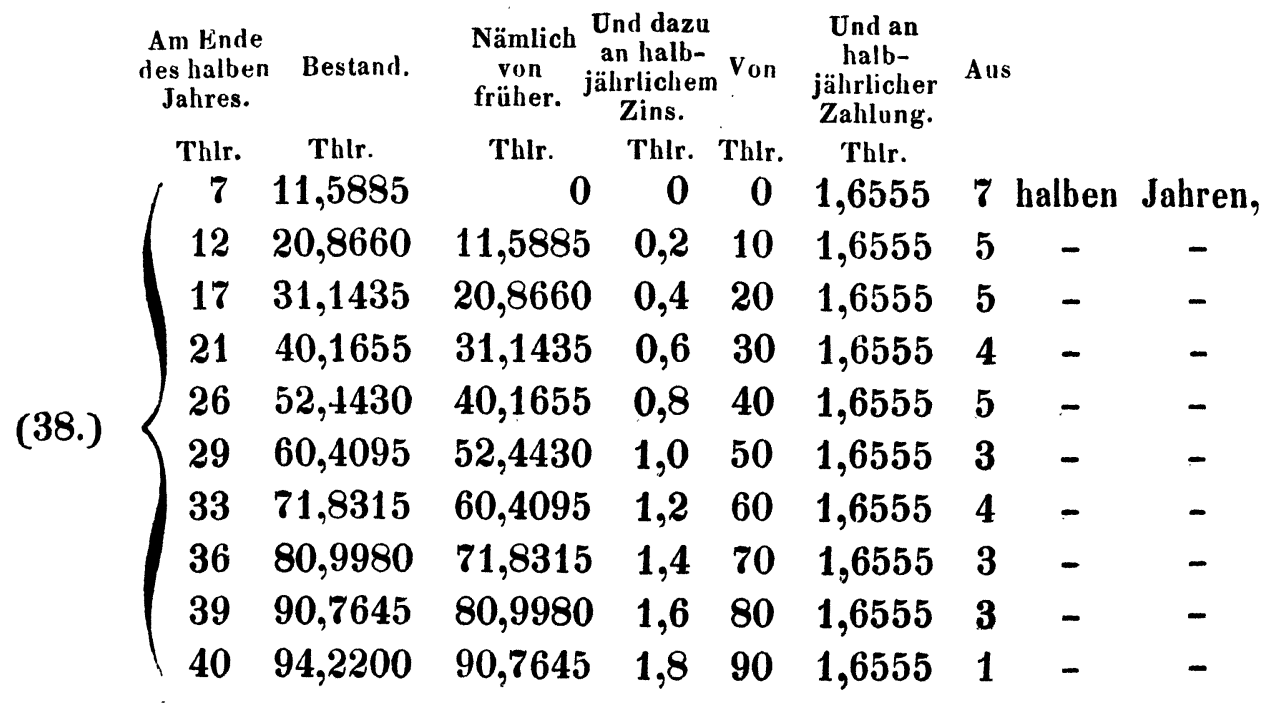


Also vermag $\boldsymbol{B}$ bis zum Ende des 20ten Jahres aus der halbjährlichen Zahlung von 1,6555 Thlrn. nur die Summe

(39.) $\quad y_{n}=94,2200$ Thlr.

anzuhäufen, folglich um beinahe 6 v. H. weeniger als ihm zukommt.

$f$. Für grö/sere Summen sind die Abweichungen, wenn man blofs einfache Zinsen in Anrechnung bringt, ebenfalls bedeutend, aber wenn man Zinseszinsen ansetzt, nur gering.

Es sei z. B.

$$
\text { (40.) } s=10000 \text { Thlr., }
$$

so ist nach (10. u. 14.), wie aus (33. u. 34.) erhellet,

(41.) $\quad \boldsymbol{Y}=179,85$ Thaler für einfache Zinsen, und

(42.) $y=165,55$ Thaler für Zinseszinsen.

g. Die Berechnung ist der obigen ganz ähnlich, weshalb sie, um den Raum zu sparen nicht hergesetzt wird. Die Ergebnisse sind:

$$
\begin{array}{ll}
\text { (43.) } & Y_{n}=10857,50 \text { Thaler, } \\
\text { (44.) } & y_{n}=9994,80 \text { Thaler. }
\end{array}
$$

Mithin bekommt $\boldsymbol{B}$, wenn man ihm blofs einfache Zinsen anrechnet, durch die halbjährliche Zahlung von 179,85 Thlrn. nach 20 Jahren 857,50 Thlr. oder über $8 \frac{1}{2}$ v. H. zu viel; wenn man ihm dagegen Zinseszinsen in Ansatz bringt nur 5,2 Thlr. zu weniy; was nicht bedeutend ist.

7.

Lautet das kleinste kăufliche Zinspapier auf mehr als 10 Thlr., was leicht sein kann, da die kleinen Stücke nicht so leicht und nicht jederzeit zu haben sind, so ist der Unterschied für kleine Summen verhältnifsmäfsig sehr bedeutend.

Ist man z. B. auf Eisenbahnprioritäts-Actien beschränkt, die gewölunlich auf

$$
\text { (45.) } \quad a=100 \text { Thlr. }
$$

lauten, so würden Dem, welcher in 20 Jahren 100 Thlr. aufhäufen soll, am Anfange der 20 Jahre weder die ihm für den Ansatz von blofs einfachen Zinsen berechneten $\boldsymbol{X}=\mathbf{5 5 , 5 5 5}$ (20.), und noch weniger die ihm für Zinseszinsen berechneten $x=45,289$ Thlr. (21.) genügen. Er würde, sobald er im Geringsten weniger als 100 Thlr. empfängt, streng genommen, gar nichts an Zinsen aufsammeln können. Da er aber wieder, wenn man ihm die vollen 100 Thlr. 
gäbe, zu viel bekommen würde, nämlich die 80 Thlr. Zinsen von 100 Thlrn. in 20 Jahren, so ist in diesem Fall die Auseinandersetzung nicht anders möglich, als dafs man ihm blofs einfache Zinsen anrechnet, also 55,555 Thlr. zahlt und nun voraussetzt, er werde Jemand finden der diese Summe zu 4 v. H. geliehen verlangt. Hier mu/s also nothwendig nach einfachen Zinsen gerechnet werden. Auch weder die halbjährliche Zahlung von 1,7985 Thlr. für einfache, noch die von 1,6555 Thlr. für Zinseszinsen berechnet, genügt, weil sich nichts davon auf Zinsen anlegen läfst. Es müssen halbjährlich $2 \frac{1}{2}$ Thlr. gezahlt werden, woraus sich dann in 40 halben Jahren die 100 Thlr. aufsammeln lassen.

Für grofse Summen ist es anders. Z. B. aus der für $s=10000$ Thlr auf Zinseszinsen berechneten Summe von 45238,9 Thlrn. (28.) lassen sich in 20 Jahren, selbst wenn $a=10$ Thlr. ist, auf die obige Weise berechnet, 9946 Thlr. aufhäufen, und aus der auf Zinseszinsen berechneten halbjährlichen Zahlung von 165,55 Thlr. (42.) noch immer 9952 Thlr., so dafs in dem einem und dem andern Falle nur etwa $\frac{1}{2}$ v. H. an der richtigen Summe fehlt.

8.

Es würde sich fragen, wie die Summe $\sigma$ (\$. 3.), aus welcher, und die halbjährliche Zahlung, durch welche sich in $n$ halben Jahren die bestimmte Summe $s$ für bestimmte $a$ und $z$ durch Hinzuthun der wirklich zu erlangenden Zinsen aufhäufen läfst, zu finden sei.

\section{Die Summe o}

läfst sich ohne Proben, und im Allgemeinen genau, in gewissen Ausnahmsfällen aber für ein nahe kommendes $s$, wie folgt berechnen.

a. Es sollen

(46.) $\sigma_{n}, \sigma_{n-1}, \sigma_{n-2}, \sigma_{n-3}, \ldots$ die Beträge bezeichnen, welche am Ende des $n$ ten, $n-1$ ten, $n-2$ ten, $n-3$ ten, etc. halben Jahres aus der gesuchten Summe $\sigma$ durch wirklich zu erlangen gewesene Zinseszinsen sich haben aufhäufen lassen; so dafs $\sigma_{n}$ die Summe $s$ selbst sein soll.

(47.) $k_{n-1}, k_{n+2}, k_{n+3}, \ldots$ sollen die grö/sten ganzen Zahlen der in $\sigma_{n-1}, \sigma_{n-2}, \sigma_{n-3}, \ldots$ enthaltenen Vielfachen des kleinsten Betrages $a$ sein, welcher sich zinsbar anlegen läfst.

(48.) $r_{n-1}, r_{n-2}, r_{n-3}, \ldots$ sollen die Reste sein, welche $k_{n-1} a, k_{n-2} a$, $k_{n-3} n, \ldots$ von $\sigma_{n-1}, \sigma_{n-2}, \sigma_{n-3}, \ldots$ übrig lassen, so dafs

(49.) $\sigma_{n-1}=k_{n-1} a+r_{n-1}, \sigma_{n-2}=k_{n-2} a+r_{n-2}, \sigma_{n-3}=k_{n-3} a+r_{n-3}, \ldots$ ist. 
b. Nun lassen sich aus $\sigma_{n-1}, \sigma_{n-2}, \sigma_{n-3}, \ldots$ für das nächste balbe Jahr nur von den Theilen $k_{n-1} a, k_{n-2} a, k_{n-3} a, \ldots$, nicht von den Resten $r_{n-1}, r_{n-2}, r_{n-3}, \ldots$, Zinsen erlangen; denn die $r$ sind kleiner als $a$ und müssen also zinslos bis auf Weiteres aufbewahrt werden. Also geben die $\sigma_{n-1}, \sigma_{n-2}, \sigma_{n-3}, \ldots$ je für das nächste halbe Jahr die Beträge

(50.) $\sigma_{n}=\sigma_{n-1}+z k_{n-1} a, \sigma_{n-1}=\sigma_{n-2}+z k_{n-2} a, \sigma_{n-2}=\sigma_{n-3}+z k_{n-3} a$, $\sigma_{n-3}=\sigma_{n-4}+z k_{n-4} a, \ldots$

c. Setzt man hierin die Ausdrücke von $\sigma_{n-1}, \sigma_{n-2}, \sigma_{n-3}, \ldots$ aus (49.), so erhălt man:

und dann aus (49.)

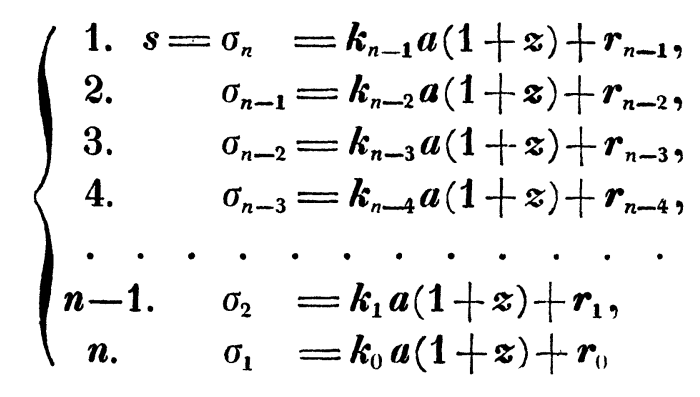$$
\text { (52.) }\left\{\begin{array}{cc}
1 . & \sigma_{n-1}=k_{n-1} a+r_{n-1}, \\
2 . & \sigma_{n-2}=k_{n-2} a+r_{n-2}, \\
3 . & \sigma_{n-3}=k_{n-3} a+r_{n-3}, \\
4 . & \sigma_{n-4}=k_{n-4} a+r_{n-4}, \\
\cdot . & \cdot \cdot \cdot \cdot \cdot \cdot \cdot \cdot \cdot \\
n-1 . & \sigma_{1}=k_{1} a+r_{1} \text { und } \\
n . & \sigma_{0}=k_{0} a+r_{0}=\sigma .
\end{array}\right.
$$

d. Man mufs also zuerst nach (51.1.) $s=\sigma_{n}$ mit $a(1+z)$ dividiren, was $k_{n-1}$ und $r_{n-1}$ und darauf aus (52.1.) $\sigma_{n-1}$ giebt. Hierauf dividirt man nach (51.2) das gefundene $\sigma_{n-1}$ mit $a(1+z)$, was $k_{n-2}$ und $r_{n-2}$ und dann aus (52. 2.) $\sigma_{n-2}$ giebt. Und so weiter bis $\mathrm{zu} \sigma_{0}=\sigma$ hinunter; welches also dann so aus $s$ gefunden wird.

e. Offenbar findet sich durch diese Rechnung das gesuchte $\sigma$ aus dem bestimmten $s$ völlig genau; aber nur dann, wenn alle Reste $r$, auf welche man kommt, kleiner als $a$ sind. Es kann aber sein, dafs man auf Reste $r$ kommt, welche gleich a und selbst grö/ser als a sind, weil die Gleichungen (51.), aus welchen man die $r$ durch die Division, nicht mit $a$, sondern mit $a(1+z)$ nimmt, nur $r$ geben, die nothwendig kleiner als $a(1+z)$, 
nicht nothwendig kleiner als $a$ sind. Ergiebt sich nun ein $r=a$ oder $>a<a(1+z)$, so läfst sich deshalb nicht etwa ein um 1 gröfseres $k$ setzen; denn dadurch würde das $r<a(1+z)$ um $a(1+z)$ kleiner und folglich negativ werden; was nicht sein darf, weil es bedeuten würde, dafs zu dem Bestand für die weitere Zeit zugelegt werden soll; wozu der Inhaber des Bestandes nicht verbunden ist. Man mufs also die $r$, welche, obgleich kleiner als $a\left(1+z^{2}\right)$, gleich oder gröfser als $a$ sind, zulcssen, und setzen, dafs sie ebenfalls zinslos bleiben; woraus sich dann aus dem gefundenen $\sigma$ rückwärts ein $\sigma_{n}$ ergeben mufs, welches um etwas grö/ser als $s$ ist. Indessen ist dieser kleine Überschufs in diesen Ausnahmsfällen nicht unbillig. Ein Überschufs über $s$ wäre sogar überhaupt in allen Fällen nicht unbillig; als einige Vergũtigung für die Mühe des Aufsammelns.

\section{Die halbjährliche Zahlung $\varepsilon$,}

welche durch die wirklich zu erlangenden Zinsen in $n$ halben Jahren die Summe $s$ einträgt, läfst sich nicht durch ein ähnliches Verfahren wie in (I.) finden, sondern nur näherungsweise, durch Proben. Denn hier würde in den Gleichungen (50.u. 51.) rechterhand $\varepsilon$ hinzukommen und es würde z. B. in (51.)

(53.) $s=\sigma_{n}=k_{n-1} a(1+z)+r_{n-1}+\varepsilon$

sein, woraus sich $k_{n-1}$ und $\boldsymbol{z}_{n-1}$ nicht finden lassen, da $\varepsilon$ noch unbekannt ist.

9.

In der Regel wird man, um $\varepsilon$, so wie auch $\sigma$ zu finden, am besten thun und am schnellsten zum Ziele kommen, wenn man erst $x$ und $y$ nach (6. u. 14.) berechnet, da die Anrechnung von Zinsessinsen, wie aus dem Obigen erhellet, im Allgemeinen das Richtige näher trifft als die Anrechnung von blofs einfacken Zinsen. Hierauf berechne man wie oben die Summen $x_{n}$ und $y_{n}$, welche sich aus den gefundenen $x$ und $y$ durch wirklich zu erlangende Zinsen aufhäufen lassen und welche stets kleiner sein werden als s. Dann, um dem richtigen gröfsern $\sigma$ und $\varepsilon$ näher zu kommen, vergröfsere man $x$ und $y$ verhältni/smä/sig, indem man nämlich

$$
\text { (54.) } \quad \stackrel{1}{x}=x \cdot \frac{s}{x_{n}} \text { und } \quad \stackrel{y}{y}=y \cdot \frac{s}{y_{n}}
$$

selzt. Man berechne nun von Neuem die Summen $\dot{x}_{n}$ und $\dot{y}_{n}$, welche aus $\dot{x}$ und $\dot{y}$ durch wirklicle zu erlangende Zinsen sich aufhäufen lassen. Treffen die $s$ noch nicht so nahe, als zu verlangen ist, so wiederbole man das Ver- 
fahren und setze

$$
\text { (55.) } \quad \stackrel{2}{x}=\stackrel{1}{x} \cdot \frac{s}{x_{n}} \text { und } \quad \stackrel{2}{y}=\frac{1}{y} \cdot \frac{s}{y_{n}} \text {. }
$$

U. s. w. bis das $s$ nahe genug erreicht ist, z. B. durch ${ }^{m}$ und ${ }^{m}$. Dieses $\stackrel{m}{x}$ und $\stackrel{m}{y}$ kann alsdann

gesetzt werden.

$$
\text { (56.) } \stackrel{m}{x}=\sigma \text { und } \stackrel{m}{y}=\varepsilon
$$

Zum Beispiel für $s=100$ Thlr., $z=0,02, n=40$ wäre nach (21.) $x=45,289$ Thlr. und nach (25.) $x_{n}=93,889$ Thlr. Ferner wäre nach (34.) $y=1,6555$ Thlr. und nach (39.) $y_{n}=94,2200$ Thlr. Also würde man nach (54.)

$$
\begin{aligned}
& \text { (57.) } \quad \dot{x}=45,289 \cdot \frac{100}{93,889}=48,236, \\
& \text { (58.) } \quad \dot{y}=1,6555 \cdot \frac{100}{94,2200}=1,7565
\end{aligned}
$$

zu setzen haben. Berechnet man nun hieraus wie in (24. und 38.) $\dot{x}_{n}$ und $\dot{y}_{n}$, so ergiebt sich dafür

$$
\text { (59.) } \quad \dot{x}_{n}=100,236 \text { und } \dot{y}_{n}=100,86 \text {. }
$$

Dies Beides kommt $s=100$ schon hinreichend nahe, und es wird daher, ganz angemessen, weil $\dot{x}_{n}$ und $\dot{y}_{n}$ etwas grö/ser als $s$ sind, (60.) $\sigma=\stackrel{1}{x}=48,236$ Thlr. und $\varepsilon=\stackrel{1}{y}_{n}=1,7565$ Thlr. gesetzt werden können.

Für gröfsere Summen z. B.: $s=10000$ Thlr., wo nach (31.) $x_{n}=9993,3$ Thaler und nach (44.) $y_{n}=9994,8 \mathrm{Thlr}$, also schon dem $s$ ziemlich nahe sich ergeben haben, ist die Annäherung nach (\$. 4.) noch passender.

10.

Es zeigt sich nun aus allem Obigen thatsächlich, dafs weder die Anrechnung von einfachen Zinsen, noch die von Zinseszinsen Das giebt, was durch die Aufsammlung von Zinsen in der Wirklichkeit zu erlangen möglich ist. Blofs einfache Zinsen gerechnet, giebt für den Aufsammler in der Regel zu viel, Zinseszinsen geben zu weniy. Für kleine Summen $s$ ist die Abweichung verhältni/sınä/sig bedeutend; je gröfser $s$, je geringer ist sie. 
Daraus folgt dann, dafs das Gesetz für dergleichen Zinsrechnungen weder, den Ansatz von blofs einfachen Zinsen, noch den Ansatz von Zinseszinsen vorschreiben darf. Durch die Vorschrift der Anrechnung von blofs einfachen Zinsen wird es den Aufsammler begünstigen, durch die des Ansatzes von reinen Zinseszinsen benachtheiligen. Das Gesetz mufs, damit es gerecht sei, vorschreiben:

Dafs bei dergleichen Zinsrechnungen nur diejenigen Zinsen und Zinseszinsen in Ansatz gebracht werden dürfen, welche sich wirklich erlangen lassen. $\mathbf{Z u}$ dem Ende mu/s es die kleinsten runden Summen bestimmen, welche sich stets und ohne namhafte Mühe für den Aufsammler zu diesem oder jenem Zinsfu/s sicher sinsbar anlegen lassen.

Und danach mufs dann, wie oben auseinandergesetzt, gerechnet werden.

11.

Andererseits folgt aus dem Umstande, dafs, wie sich oben zeigte, Das, was sich wirklich erlangen läfst, von dem nach reinen Zinseszinsen Berechneten für gro/se Summen verhältnifsmäfsig nur unbedeutend abweicht, dafs eine Sparcasse, wie die in (Band 39. S. 183) beschriebene, die mit Hunderttausenden und Millionen von Thalern zu verkehren haben würde, unbedenklich nach reinen Zinseszinsen rechnen darf; wie es auch dort geschehen ist. Der kleine Verlust wird durch den möglichen Gewinn beim Ankauf der Papiere vielfach gedeckt. Was aber eine Casse mit der grofsen Summe vieler vereinigten Summen zu thun vermag, das vermag nicht der Einzelne; am wenigsten mit einer kleinen Summe.

Ferner folgt, dafs, wenn eine Sparcasse wie die gemeinte vorhanden wäre, dadurch auch alle Schwierigkeiten und mögliche Streitigkeiten bei den Zinsrechnungen vermieden werden würden. Denn wenn z. B. Jemand die Summe $s$ nach $n$ halben Jahren zu empfangen hätte, so könnte er, wenn er Dasjenige sogleich verlangt, woraus er in $n$ halben Jahren die Summe $s$ durch die Zinsen aufzuhäufen vermögen würde, ohne Weiteres durch diejenige Summe in Sparcassenscheinen befriedigt werden, die nach $\boldsymbol{n}$ halben Jahren die Summe $s$ werth ist; und das um so mehr, da ihm dann zugleich alle Mühe und Wagnifs beim Aufsammeln erspart wird. Die Sparcasse übernimmt diese Mühe und dieses Wagnifs für ihn. Das Gesetz könnte ihn sogar geradezu 
auf die Sparcasse verweisen; etwa neben der obigen Bestimmung. Daraus folgt ebenfalls noch ein Nutzen der vorgeschlagenen Sparcasse.

Diese Bemerkung ist der in der Überschrift gedachte Nachtray, welcher noch zu der genannten Abhandlung über Sparcassen nothwendig war.

12."

Es möge noch einer Art von Zinsrechnung gedacht werden, welche zwar in der Wirklichkeit nicht vorkc mmt, aber theoretisch nicht ohne Interesse ist.

a. Es ist hier oben überall angenommen worden, dafs die Zinsen halbjührlich gezahlt werden. Aber es sind auch Zinsen möglich, die vierteljührlich, monatlich, wöchentlich, täglich und in beliebig noch kleineren Theilen des Jahres zahlbar sind. Geht man bis zur Theilung des Jahres in unendlich viele Theile, so würde dies Zinsen geben, die sich stetig (continuirlich) nennen lassen, und es früge sich, welches der Ertrag solcher Zinsen sein würde.

b. Theilt man das halbe Jahr und auch den halbjährlichen Zins in $m$ Theile, so würde, wenn $z$ wie oben den halbjährlichen Zins von 1 bezeichnet, in jedem Zeittheil $\frac{1}{m}$ die Summe $1,1+\frac{z}{m}$ mal so grofs werden, also würde 1 in $n$ halben Jahren bis auf

$$
\begin{gathered}
\text { (61.) } \quad s_{1}=\left(1+\frac{z}{m}\right)^{n m} \\
=1+n m \cdot \frac{z}{m}+\frac{n m(n m-1)}{2} \cdot \frac{z^{2}}{m^{2}}+\frac{n m(n m-1)(n m-2)}{2.3} \cdot \frac{z^{3}}{m^{3}} \ldots \ldots
\end{gathered}
$$

anwachsen. Dies giebt für $m=\infty$,

$$
\text { (62.) } \quad s_{1}=1+n z+\frac{n^{2} z^{2}}{2}+\frac{n^{3} z^{3}}{2.3} \cdots
$$

Die Reihe rechts ist nichts anderes als $e^{n z}$, wo

$$
\text { (63.) } \quad e=2,7182818285
$$

und die Grundzahl der natürlichen Logarithmen ist. Also ist

$$
\text { (64.) } s_{1}=e^{n z} \text {. }
$$

c. Theilt man den halbjährlichen Zins $z$ nicht in $m$ Theile, wie das halbe Jahr, sondern rechnet auf den Zeittheil $\frac{1}{m}$ so viel, dafs in $m$ Zeittheilen oder in einem halben Jahre die Summe 1 gerade auf $1+z$ anwächst, so 
kommt man natürlich auf die gevöhnliche Zinseszinsrechnung und es ist (65.) $\quad s=(1+z)^{n}=1+n z+\frac{n(n-1)}{2} z^{2}+\frac{n(n-1)(n-2)}{2.3} z^{3} \ldots$

d. Für $n=1$ ist

$$
\text { (66.) } \quad s_{1}=e^{z} \text { und } s=1+z \text {. }
$$

Dies würde für $z=0,02$,

$$
\text { (67.) } s_{1}=1,02022 \text { und } s=1,02
$$

geben. Für $n=40$ und $z=0,02$ wären

$$
\text { (68.) } s_{1}=2,22553 \text { und } s=2,20805 \text {. }
$$

Die Unterschiede sind, wie man sieht, nicht bedeutend. Berlin, im December 1852. 